\title{
Image Retrieval by Soft Computing Technique and Visual Features
}

\author{
Ashwani Mathur \\ IT Consultant, Senior Manager
}

\begin{abstract}
Digital platform-based services increase content on servers and retrieval of relevant information depends on data matching algorithms. Out of different type of data image plays a crucial role for various document proof, study, analysis, diagnosis. Hence retrieval of relevant image as per requirement is very important. This paper has proposed an image retrieval model which takes visual, text query as input and provide relevant images. Work has utilized soft computing genetic algorithm technique for the initial image storage in clusters. Genetic algorithm finds the cluster center images as per visual feature know as co-occurrence matrix and text query. Cluster center images cluster whole image dataset and act as filter to extract relevant image as per user query. Implementation of proposed work was done on Matlab and experiment was performed on real image dataset. Result shows that proposed model has increase the image relevancy as per user requirement.
\end{abstract}

\section{Keywords}

Genetic Algorithm, Image retrieval, Soft Computing, Visual Feature Extraction

\section{INTRODUCTION}

Image from electronic camera, satellite, medical instrument, etc. have different role as per type of requirement. Life of data depends on storage and extraction methods; hence computer algorithm plays a significant role. Out of different data types most unorganized data is image as relevancy between image is depends on manual memory. So, indexing of images by manual operation is time taken, costly, method for a dynamic and bulky data. Images are store by some keyword or annotations as well, but it was found that visual content are different for same set of keywords [1]. This limitation was resolved by visual feature extraction from the image. Content based image/picture recovery also known as pass by example query. Content based means that the explore analyzes the content of the image rather than the metadata such as keyword tags or explanations associated with the image [2].

The accessibility of image/picture known as characteristics. The availability of image/picture capturing gadgets such as digital camera, image scanners, and the volume of digital image set is increasing quickly. It is essential to resourcefully store and reclaim image for diverse application such as fashion design, crime prevention, medicine, architecture [3]. CBIR is image have affluent content. This content can be taken out as various content features. It takes the accountability of forming the question away from the user and each image/picture will now explain by its own functions.

Mismatching of data of image query data and the stored image statistics in the dataset is attainable. This selected gape to match the image/picture on the likeness basis is called semantic gap. Users insert some queries for which optical likeness does not match entirely with human observation. By which a semantic gap between CBIR system and the user is obtained $[4,5]$. Semantic recovery has some boundaries. A complexity present in it is that the majority of the images have more than one semantic understanding. Because images used for training have usually short explanation in form of a caption, therefore, some features might never be known. This helps to diminish the amount of images occasions used for training and deteriorates the system's ability to be trained for the concepts that are unusual and which have a high changeable visual appearance. Semantic retrieval system has a narrow vocabulary, hence mixture of visual and semantic was planned in this work as done in other set of papers [6].

\section{RELATED WORK}

Chintamani Chavan et al. [7] conversed a method that provided Gabor's Magnitude Fusion and Modified Block Truncation Coding utilizing cloud computing by retrieving content-based images (CBIR). It is an open source cloudbased computer system. Here, the SaaS architecture of CBIR has been projected because the services are made obtainable vigorously and result in an augment in the scalability, elasticity and accessibility of the applications. The major reason for this CBIR system is to give the finest matches and find images in great databases using their content as low-level descriptors. According to this system, the cloud services projected by the cloud architecture will handle all the unanticipated traffic, and at the same time, they will assistance from a minimized cost. This open source project can be enhanced upon request and incorporated into other accessible systems.

Stanisław Deniziak et al. [8] addressed a new technique for image recovery that is based on two thoughts: an object demonstration and a matching algorithm. Here, the new CBIR algorithm that utilizes the inquiry by estimated form was presented. A technique was projected that is based on the disintegration of forms into smaller, primitive segments, which are explained by their attributes. Based on the primitives detected, a graphic representation of the form is constructed, and then compared with the graphs accumulated in the datasets. The major benefit of this approach is that it can be applied to transformed covered objects or partly covered objects. This algorithm is appropriate for queries that utilize the key image, as well as for queries drawn by man. The course of future research is the well-organized storage of graphics of objects in the record.

Chunheng et. al. in [9] writer suggested a straightforward but effectual semantic-based aggregation (SBA) technique. The projected SBA used the discriminative filters of profound convolutional layers as semantic detectors. Furthermore, suggest the effectual unverified scheme to choose several semantic detectors to produce the soft region proposals, which 
emphasize convinced discriminative guide of objects and suppress the sound of background. Identifying CNN based image pattern increase the execution time while security of data was not involved in this work.

Aasia Ali in the. [10] author utilized the SIFT feature of image for retrieval of relevant content. Visual SIFT feature were pass in deep neural network for training and it was obtained that resultant trained neural network was better as compared. Paper has further improved the work performance by involving the text phrases as well.

Jiaohua et. al. in [11] has extract the corner feature from the image which are some points in the image and based on similarity of those points a has index was prepared by the authors. Paper has annotation feature as well for increasing the accuracy of the work as visual feature corner was quit a weak feature point collection.

\section{PROPOSED METHODOLOGY}

Explanation of proposed model is done in this section, where fig. 1 represent block diagram with explanation of model. Here whole working is divided into two section first for dataset learning where images were features were extract and arrange in hieratical structure. While second was testing module where similar features were extract from it and compare with learned model for image extraction.

\section{Pre-Processing}

Input images from the dataset are of different dimension and have different format as well. So, each image needs to be resized into fix dimension which help in matrix operations, input image has annotations as well which will be pre-process by removing special characters as well.

\section{Feature Extraction}

In this work two type of features were used for the image retrieval first was visual where CCM values were obtained. Input color image was transformed into RGB (Red, green, Blue) and HSV (Hue, Saturation, Value) format. Matrix from $\mathrm{R}, \mathrm{G}, \mathrm{H}$ and $\mathrm{S}$ were pass in Eq. 1, 2, 3 and 4. Output of this feature set is sixteen value set for a single image.

$$
\begin{gathered}
\text { InverseDifference }=\sum_{i=1} \sum_{j=1} \frac{1}{\left(1+(i-j)^{2}\right.} m(i, j)--E q .1 \\
\text { Entropy }=-\sum_{i=1} \sum_{j=1} m(i, j) \log [m(i, j)]--E q .2 \\
\text { Energy }=\sum_{i=1} \sum_{j=1}(m(i, j))^{2}--E q .3 \\
\text { Contrast }=\sum_{i=1} \sum_{j=1}(i-j)^{2} * m(i, j)--E q .4
\end{gathered}
$$

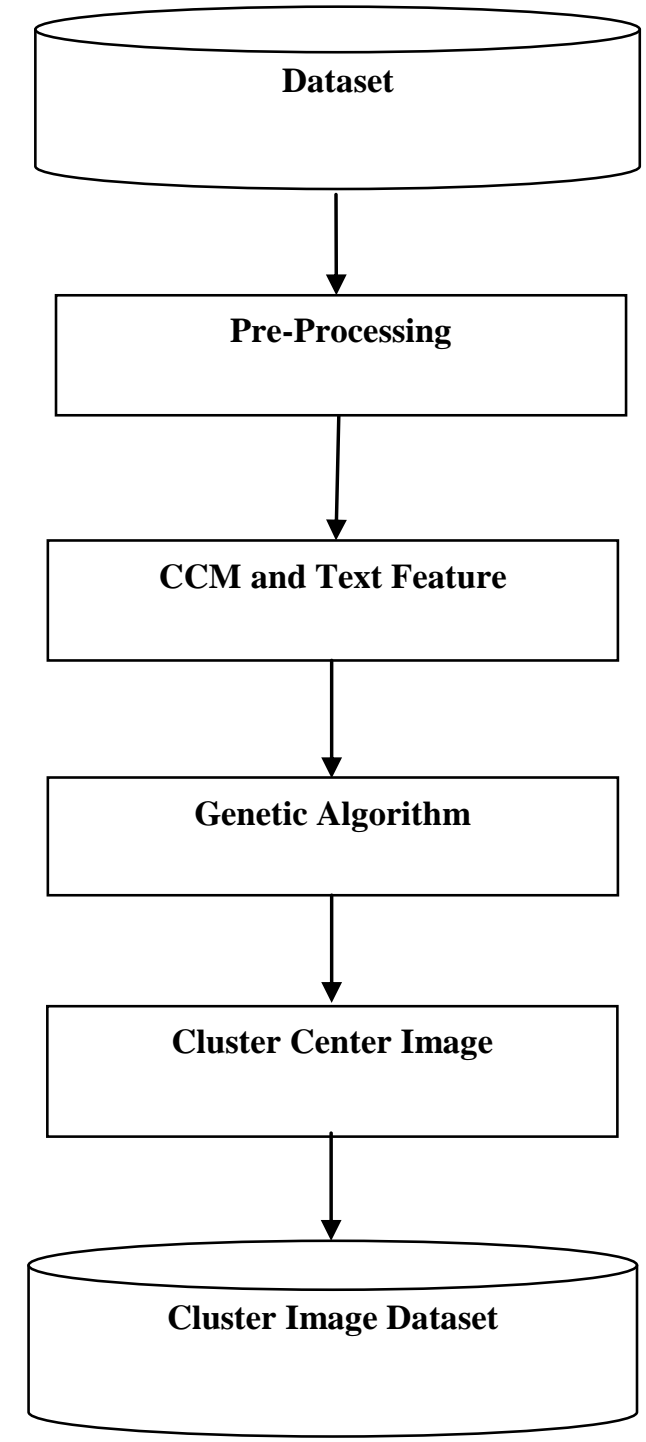

Fig. 1 Proposed image retrieval algorithm

Further each annotation string is converted into set of words as per text pre-processing, so similar kind of image may have different set of words. This annotation increase searching accuracy. Hence feature set is collection of two type of data first is CCM and second was words from annotation.

\section{Feature $\leftarrow[\mathrm{CCM}$, Annotations $]$}

\section{Genetic Algorithm}

In this image retrieval model images obtained from the dataset were arrange into clusters. So, representation of cluster was done by few sets of images hence identification of those images from available set is done by this genetic algorithm. In this algorithm updating of population was done twice in single iteration by different crossover approaches.

\section{Generate population}

As population is collection of chromosomes where each chromosome is collection of cluster centers. Hence chromosome having set of image features which act as cluster center. To better understand this let image dataset have $\mathrm{N}$ number of images so one of possible solution is Chromo $=$ $\left\{\mathrm{C}_{1}, \mathrm{C}_{5}\right\}$. In similar way other set of images were collect form dataset randomly shown in table 1. 
Table 1. Chromosome set

\begin{tabular}{|l|l|}
\hline $\mathrm{C}_{1}$ & $\mathrm{C}_{5}$ \\
\hline $\mathrm{C}_{3}$ & $\mathrm{C}_{4}$ \\
\hline $\mathrm{C}_{1}$ & $\mathrm{C}_{2}$ \\
\hline $\mathrm{C}_{2}$ & $\mathrm{C}_{6}$ \\
\hline
\end{tabular}

\section{Fitness Function}

On the basis of comparison similarity between the annotation and text annotation is found. So number of same keywords are consider as the similarity measure for filtering the image dataset on the basis of textual feature of first chromosome $\left[\mathrm{C}_{1}\right.$ $\left.\mathrm{C}_{5}\right]$.

Table 2 Image annotation example

\begin{tabular}{|c|c|c|c|}
\hline $\begin{array}{l}\text { Image } \\
\text { Name }\end{array}$ & Annotations & $\begin{array}{l}\text { Text } \\
\text { Query }\end{array}$ & $\begin{array}{c}\text { Text } \\
\text { Similarity } \\
\text { (TS) }\end{array}$ \\
\hline I1 & $\mathrm{a} 2, \mathrm{a} 6, \mathrm{a} 7, \mathrm{a} 4 \mathrm{a} 1$ & \multirow{3}{*}{$\begin{array}{c}\text { a1, a2, a3 } \\
\text { a4, a5 }\end{array}$} & 3 \\
\hline $\mathrm{I} 2$ & $\mathrm{a} 8, \mathrm{a} 2, \mathrm{a} 1 \mathrm{a} 4 \mathrm{a} 2$ & & 2 \\
\hline I3 & $\mathrm{a} 1 \mathrm{a} 2, \mathrm{a} 9 \mathrm{a} 10, \mathrm{a} 9$ & & 2 \\
\hline
\end{tabular}

\section{Visual Feature distance}

Let understand by subtracting (Euclidian distance) the CCM features of image 1 and 2 .

Table 3 Image CCM Feature value.

\begin{tabular}{|l|lclllllllll|}
\hline I1 & 4 & 23 & 18 & 24 & 23 & 41 & 33 & 29 & 23 \\
& 21 & 8 & 7 & 2 & 0 & 0 & 0 & & & \\
\hline I2 & 1 & 20 & 16 & 24 & 23 & 41 & 33 & 29 & 19 \\
& 21 & 4 & 4 & 5 & 4 & 4 & 2 & & & \\
\hline Subtraction & 3 & 3 & 2 & 0 & 0 & 0 & 0 & 0 & 4 \\
& 0 & 4 & 3 & 3 & 4 & 4 & 2 & & & \\
\hline Summation & 32 & & & & & & & & & \\
\hline
\end{tabular}

\section{$\mathrm{F}=(1 / \operatorname{Min}($ Euclidian $(\mathrm{ID}, \mathrm{PC}))) * \lambda+\operatorname{Max}(\operatorname{Similarity}(\mathrm{A}, \mathrm{PC}) * \beta$}

Now as per above distance matrix table for first chromosome, set of clusters will be 14.545 . So as per chromosomes cluster center position overall fitness value of chromosome is 14.545 . In similar fashion other set of solutions were calculate. If 14.545 is highest value among other set of chromosomes than $\mathrm{Ch}_{\text {best }}$ is 14.545. Let us consider maximum iteration value $\mathrm{M}_{\mathrm{r}}$ is 10 .

Table 4 Image fitness value

\begin{tabular}{|l|l|l|l|}
\hline Image & $\mathrm{C}_{1}$ & $\mathrm{C}_{5}$ & Image Weight \\
\hline $\mathbf{I}_{\mathbf{2}}$ & $(1 / 32)^{*} 10+3 * 1$ & $(1 / 2)^{*} 10+1 * 1$ & $(1 / 2)^{*} 10+3 * 1=8$ \\
\hline $\mathbf{I}_{\mathbf{3}}$ & $(1 / 46)^{*} 10+2 * 1$ & $(1 / 48) * 10+1 * 1$ & $(1 / 46) * 10+2 * 1=2$. \\
& & & 217 \\
\hline $\mathbf{I}_{\mathbf{4}}$ & $(1 / 90)^{*} 10+2 * 1$ & $(1 / 94) * 10+1 * 1$ & $(1 / 90) * 10+2 * 1=2$. \\
& & & 111 \\
\hline Fitness & & 12.328 \\
\hline
\end{tabular}

\section{Crossover Operation}

Crossover as per chromosome is shown below:

\begin{tabular}{l|l|l|}
$\mathrm{Cc}_{\text {Lbest }}=$ & $\mathrm{C}_{1}$ & $\mathrm{C}_{5}$ \\
\hline
\end{tabular}

Now all other set of chromosomes are assume one of them be

$\mathrm{Cc}=$

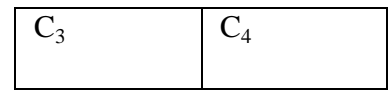

So, let $\mathrm{i}$ position in the vector is 2 than at second position $\mathrm{Cc}_{2}$ chromosome value is replace by $\mathrm{Cc}_{\text {Lbest, } 2}$ value, hence new chromosome is

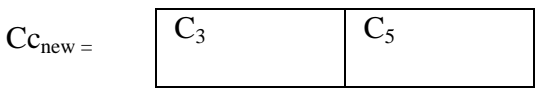

Now population after crossover will be:

\begin{tabular}{|l|l|}
\hline $\mathrm{C}_{1}$ & $\mathrm{C}_{5}$ \\
\hline $\mathrm{C}_{3}$ & $\mathrm{C}_{5}$ \\
\hline $\mathrm{C}_{1}$ & $\mathrm{C}_{5}$ \\
\hline $\mathrm{C}_{1}$ & $\mathrm{C}_{6}$ \\
\hline
\end{tabular}

In similar fashion other set of group chromosomes were modified, here it is possible that modification of chromosome was done at more than one place. Now check for maximum iteration value, if reach than find best chromosome and classify image.

\section{Cluster Dataset}

Genetic algorithm gives an output after maximum number of iterations or when two iteration gives same chromosome as best solution. Hence as per best chromosome cluster center other set of non-cluster center images were grouped by using both type of feature value.

\section{Testing Phase}

Once dataset get grouped into cluster form than testing dataset will pass and evaluate the resultant ranked images. So, each image from the testing dataset is pre-process first as done in learning phase, further similar visual, annotation features were also extract. Finally based on testing image feature cluster center feature values were compared and most matching cluster is select for the image ranking. Now each clustered image features were compared with testing image feature for final rank of images. This comparison was done by fitness function.

\section{EXPERIMENT AND RESULT Experimental Setup}

Proposed image retrieval model of genetic algorithm and CCM feature was developed on MATALB software. Real dataset (http://wang.ist.psu.edu/d0cs/related.shtml) of four different categories was used for comparison of proposed model with existing algorithm proposed in [11]. 


\section{Results}

Table 5 Top image retrieval-based precision value comparison

\begin{tabular}{|c|r|r|}
\hline Images & Proposed Work & \multicolumn{1}{l|}{ Previous Work } \\
\hline Person & 0.851 & 0.51 \\
\hline Construction & 0.575 & 0.306 \\
\hline Vehicle & 0.572 & 0.414 \\
\hline Creature & 0.799 & 0.463 \\
\hline
\end{tabular}

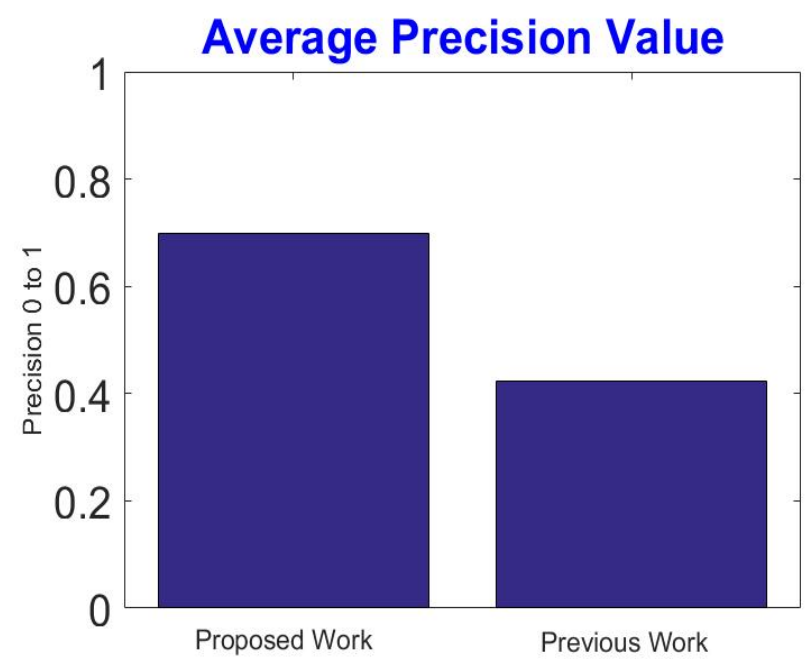

Fig. 2 Average precision value-based comparison of image retrieval models

Table 5 and fig. 2 shows precision value-based comparison of proposed genetic algorithm, previous model [11]. It was obtained that precision value of proposed model was improved by $39.47 \%$ as compared to [11]. Use of CCM feature with text-based image retrieval increased the parameter value.

Table 6 Top image retrieval based NDCG value comparison.

\begin{tabular}{|c|r|r|}
\hline Images & Proposed Work & \multicolumn{1}{|c|}{ Previous Work } \\
\hline Person & 0.887 & 0.65 \\
\hline Construction & 0.607 & 0.475 \\
\hline Vehicle & 0.626 & 0.577 \\
\hline Creature & 0.890 & 0.631 \\
\hline
\end{tabular}

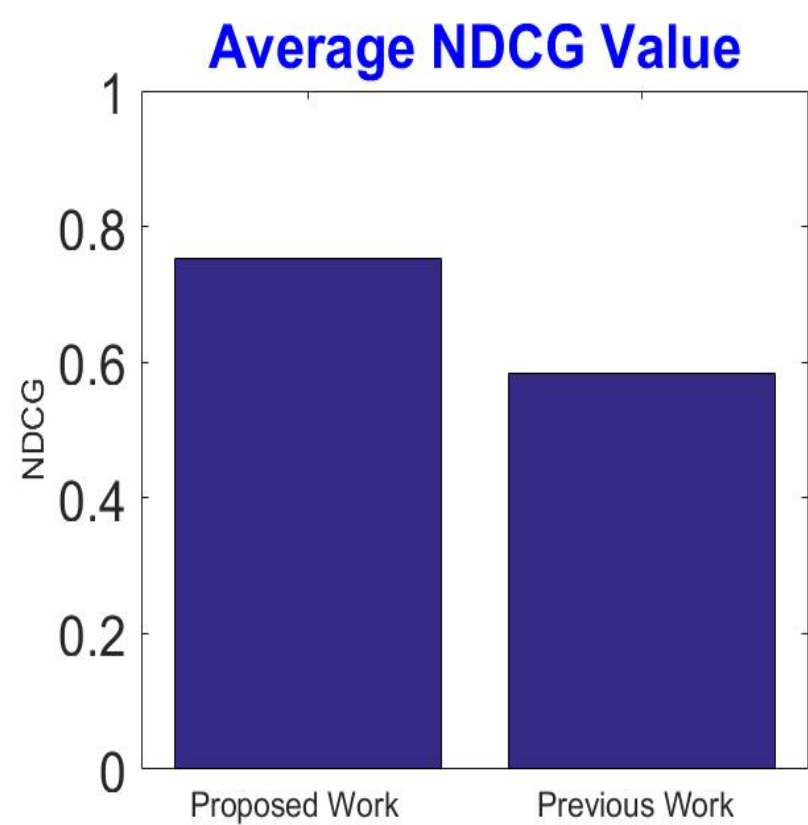

Fig. 3 Average NDCG value-based comparison of image retrieval models

Table 6 and fig. 3 shows NDCG value-based comparison of proposed genetic algorithm and previous model [11]. It was obtained that NDCG value of proposed model was improved by $22.49 \%$ as compared to [11]. Cluster center selection by genetic algorithm for image type representation improved the image retrieval NDCG evaluation parameter.

Table 7 Average Execution Time in Seconds Based Comparison of Proposed Model

\begin{tabular}{|c|c|c|}
\hline Images & Previous Work & Proposed Work \\
\hline Person & 0.863 & 0.703 \\
\hline Construction & 0.863 & 0.743 \\
\hline Vehicle & 0.863 & 0.763 \\
\hline Creature & 0.863 & 0.723 \\
\hline
\end{tabular}

Table 7 shows precision value-based comparison of proposed genetic algorithm and previous model [11]. It was obtained that execution time value of proposed model was reduced by $3.94 \%$ as compared to [11]. Use of CCM feature with textbased image retrieval reduced the execution time.

\section{CONCLUSION}

Expansion in data transmission capacity help users to upload multimedia content on servers, which raise the relevant content retrieval issue. This paper has resolved the image content retrieval method which extract CCM image feature from the user visual and text query. Proposed model cluster image dataset into cluster as per genetic algorithm cluster center images. Dynamic nature of cluster center image selection increases the relevancy of image as per user requirement. Experiment was done on real dataset images and result shows that proposed model has increase the precision value by $39.47 \%$ and NDCG value by $22.49 \%$ as compared to another existing algorithm. Researcher can adopt other soft computing algorithm for increasing the relevancy of image retrieval. 


\section{REFERENCES}

[1] Patil, P.B. and M.B. Kokare, "Relevance feedback in content-based image retrieval: A review" J. Appli. Comp.Sci. Math., 10: 41-47.

[2] Sandeep kumar, Zeeshan, Anuragjain, "A review of content-based image classification using machine learning approach", International journal of advanced computer research (ISSN (print): 2249-7277 ISSN (ONLINE): 2277-7970) volume-2 number-3 Issue-5 september-2012.

[3] Bindita Chaudhuri, Begüm Demir, Lorenzo Bruzzone, and Subhasis Chaudhuri. "Region-Based Retrieval of Remote Sensing Images Using an Unsupervised GraphTheoretic Approach". IEEE Geoscience And Remote Sensing Letters, Vol. 13, No. 7, July 2016987

[4] Wenjun Lu, Avinash L. Varna, , Min Wu Confidentiality-Preserving Image Search: A Comparative Study Between Homomorphic Encryption and DistancePreserving Randomization". Received December 15, 2013, accepted January 15, 2014, date of publication February 20, 2014, date of current version March 4, 2014.

[5] Shubhangi P. Meshrama, Dr. Anuradha D. Thakareb ,Prof. Santwana Gudadhe . "Hybrid Swarm Intelligence Method for Post Clustering Content Based Image Retrieval". 7th International Conference on
Communication, Computing and Virtualization 2016.

[6] B.Jyothi , Y.Madhaveelathaby Multidimensional Feature Space For An Effective Content Based Medical Image Retrieval, (02015 IEEE.

[7] Chintamani Chavan;Madhavan Dixit (2017): A Survey Of Content-Based Image Retrieval Using Cloud Computing. IJESC-International Journal Of Engineering Science And Computing, Volume 7, Issue 6.

[8] Stanisławdeniziak; Tomasz Michno (2016): ContentBased Image Retrieval Using Query By Approximate Shape. ACSIS: Agents For Cooperative Secured Information Systems, Volume 8.

[9] Jian Xu, Chunheng Wang, Chengzuo Qi, Cunzhao Shi, And Baihua Xiao. "Unsupervised Semantic-Based Aggregation Of Deep Convolutional Features". IEEE Transactions On Image Processing 2018.

[10] Aasia Ali; Sanjay Sharma (2017): Content-Based Image Retrieval Using Feature Extraction With Machine Learning International Conference On Intelligent Computing And Control Systems.

[11] Jiaohua Qin, Hao Li, Xuyu Xiang, Yun Tan, Wenyan Pan, Wentao Ma1, And Neal N. Xiong. "An Encrypted Image Retrieval Method Based On Harris Corner Optimization And Lsh In Cloud Computing". Ieee Access March 7, 2019. 